\title{
Belgitude as a philosophy of relation to oneself, to the other, to history and compared with the expression of sicilitude
}

\section{[La belgitude comme philosophie d'un rapport a soi, a l'autre, a l'histoire ; et confrontee a une expression de la sicilitude]}

\author{
Julien Delvaux
}

DOI: $10.18355 /$ XL.2020.13.01.21

\begin{abstract}
The notion of literary " belgitude» is particularly used since 1980. It is largely inspired by a reflection on the major federal reforms, which give Belgium the air of a country which « exists and does not exist ». In Pierre Mertens'work, it designates a condition of wandering experienced by the French-speaking writer, which implies a new relationship with oneself, with history and with the other (in particular French). This article analyzes the novel Une paix royale (1995) as a particularly representative text, and carrier material for a course on the civilization of french speaking cultures. It ends by proposing a confrontation with the sicilitude of Sciascia, which also designates a specific existential condition.
\end{abstract}

Key words: belgitude, identity in non-place, element (Bachelard's philosophy), water intertextuality, relationships between fiction and history, counterfeiter, sicilitude

\section{Résumé}

Particulièrement en vogue depuis 1980, la notion de «belgitude » littéraire s'est alimentée d'une réflexion sur les grandes réformes fédérales, qui donnent à la Belgique les airs d'un pays qui à la fois «existe et n'existe pas ». Dans l'oeuvre de Pierre Mertens, elle désigne une condition d'errance de l'écrivain francophone, qui implique un nouveau rapport à soi-même, à l'histoire et à l'autre (notamment français). Cet article analyse le roman Une paix royale (1995) en tant que particulièrement représentatif, et matériau porteur pour un cours de civilisation du français langue étrangère. Il s'achève en proposant une confrontation avec la sicilitude de Sciascia, qui désigne elle aussi une condition existentielle spécifique.

Mots clés : belgitude, identité en non-lieu, élément (philosophie de Bachelard), intertextualité de l'eau, rapports entre fiction et histoire, faussaire, sicilitude

Si la notion de belgitude apparaît déjà dans les cahiers de Jacques Brel en 1971, sa théorisation spécifique sous les traits de l'errance et d'une identité comme non-lieu émerge en 1976 avec l'écrivain Pierre Mertens et le sociologue Claude Javeau. La ligne de ces derniers allait résumer bientôt les positions singulières du collectif La Belgique malgré tout (1980), au sein duquel une pluralité d'écrivains francophones belges, désormais exposés à la restreinte étiquette de «WalloBruxellois ", s'interrogeaient sur leurs caractéristiques propres. Depuis 1970 en effet, une phase de grandes réformes de l'Etat belge, toujours à la question aujourd'hui, avait été entamée : sur la scène internationale, l'heure était au brouillard de la construction européenne et au besoin de se repositionner après l'indépendance du Congo, tandis que le plan intérieur se voyait toujours plus compromis par des énergies de dissolution (montée des voix régionalistes), en même temps que les assises économiques se trouvaient bouleversées (déclin de l'industrie wallonne). La Belgique réinventa son équilibre dans une délégation des pouvoirs aux Régions et aux Communautés, offrant l'image d'un "pays qui existe et n'existe pas". Thème inspirateur de la belgitude, et ô

XLinguae, Volume 13 Issue 1, January 2020, ISSN 1337-8384, eISSN 2453-711X 
combien d'actualité : huit mois après les élections de mai 2019, le pays témoigne d'une énième difficulté à se doter d'un gouvernement.

Cette délégation des pouvoirs allait reconfigurer entre autres les structures culturelles de l'Etat, et doter désormais chaque Communauté linguistique, la francophone comme la néerlandophone et la germanophone, de possibilités d'expression propre. Une césure dans l'histoire des lettres francophones belges allait en naître, puisque le souffle de celles-ci allait désormais concerner une Communauté et non plus l'intégrité nationale. Pour situer cette nouveauté, on peut la situer à l'intérieur d'un cadre comprenant trois grandes périodes, basées chacune sur un sens différent de la référence à Paris, et identifiables à partir de positions groupées et explicites de la part des auteurs belges (Denis et Klinkenberg, 2005).

- Dans la première d'entre elles, consécutive à la naissance de l'Etat belge en 1830 comme artifice dérivé de l'Europe post-napoléonienne, on peut souligner une recherche d'émancipation, ambigüe, par rapport aux références littéraires parisiennes. Cette volonté de se définir est identifiable aux rassemblements de la Jeune Belgique, autour desquels on entendit un jour parler d"'âme belge" (Picard), mais dont on préfère retenir cette esthétique du "mythe du nord", conçue comme un alliage spécifique de traits latins et germains.

- $\quad$ A cette phase succède une aspiration centripète (1920-1970), basée sur un rejet du trait germain au lendemain du premier conflit mondial, et explicitée dans le tardif Manifeste du groupe du lundi (1937).

- $\quad$ La troisième phase comprend entre autres la belgitude, et peut être qualifiée avec Denis et Klinkenberg (2005 : 226) de "dialectique".

Dans l'optique de situer la troisième phase, nous examinerons ici le roman Une paix royale (1995), de Pierre Mertens (1939-). Ce dernier, depuis les années 1970 , se pose en figure forte de la scène littéraire belge en même temps qu'il l'enrichit de ses activités de juriste spécialisé en droit international. Son œuvre fournit un matériau naturel pour tout cours de civilisation en français langue étrangère qui s'intéresserait à l'identité francophone belge sur fond des structures institutionnelles, et à la manière dont la littérature "parle" une région, se l'approprie, en investit l'histoire. L'écrivain incarne également un engagement intellectuel précis, soit une disposition que l'on retenait plus spontanément sous des expressions françaises depuis les Hugo et Zola. Le roman suscita diverses polémiques, ainsi qu'un procès intenté par des membres de la famille royale belge.

Cet article envisagera d'abord la définition de la belgitude comme rapport à soi, à l'autre et à l'histoire. Comme références premières à des fins didactiques, on conseillera le panorama général fourni par Quaghebeur (2006 : 281-295), l'étude du politique par Magnette (1998 : 39-48), du philosophique par Bergen (1998 : 85-100), ainsi que la lecture d'inspiration psychanalytique de Desorbay (2006 : 217-232). Pour terminer, nous envisagerons quelques principes d'une lecture croisée de thèmes entre la belgitude de Mertens et la sicilitude de Sciascia, que le premier cité rencontre explicitement (1989: 183-196) : deux voix «périphériques » définissant chacune presque deux anthropologies, en tout cas deux véritables conditions existentielles et deux rapports spécifiques à l'Histoire. L'idée de cette comparaison a fait l'objet d'exposés personnels aux Universités de Catane et d'Ostrava, et a trouvé une nouvelle stimulation dans les recherches récentes de Fichera (2017).

\section{Sur la belgitude comme identité, et le cadre théorique de son époque}

Dans la préface de La Belgique malgré tout (1980), collectif qu'il dirige, Jacques Sojcher rappelle René Char: "Épouse et n'épouse pas ta maison». Et 
Beckett : « Il est minuit. La pluie fouette les vitres. Il n'était pas minuit. Il ne pleuvait pas ». Sur cette grande terre du surréalisme et de la concorde des contraires, maintenant, « on marche sur le vide» (Collin). Sans rejoindre la convergence d'un manifeste, les écrivains du collectif prennent ensemble acte d'un " ici et ailleurs », d'une perte de patrie et de nom propre. Mais devait-ce être pour autant une impasse ? Tout au contraire, le vide allait se faire le lieu d'un pari créatif. De même que l'esthétique du mythe du nord avait sublimé, à la fin du XIXe siècle, les clivages et questions du jeune État dans des formes remarquables, l'incertitude d'un «pays qui existe et n'existe pas » et la perte d'origine deviendront des ressources inspirantes : la perte d'origine n'est pas un défaut d'origine mais justement «ce qui permet de revenir », dit Sojcher (1980 : préface). C'est donc l'errance et même le fourvoiement qui sont à créditer. D'ailleurs, comme le dit le personnage principal d'Une paix royale: "Quand on arrive effectivement là où on devait se rendre, on n'est déjà plus un voyageur, mais un simple touriste » (p. 20). Dès La fête des anciens (1971), les romans de Pierre Mertens, pour prendre l'exemple le plus caractéristique, exprimeront souvent une recherche d'unité perdue, de réconciliation avec soi-même et le monde.

Que la belgitude se définisse comme une condition d'errance en fait une identité remarquablement riche. Elle prévient notamment des récupérations idéologiques : "Constater en Wallonie la permanence d'une tradition poétique hyperclassique et prudente pour la référer ensuite à une âme wallonne revient à annihiler l'effet potentiel de la trouvaille» (Quaghebeur, $1980: 524$ ). Il ne s'agit en effet pas de circonscrire une identité en tant que substance arrêtée mais plutôt d'incarner et de peindre une position en plus d'un point comparable avec un concept d'identité tel qu'on le trouve développé dans la pensée de Deleuze (1968) : non pas l'identité grecque comme un demeurer soi-même à travers ses changements, mais ce fait de changer constamment d'avec soi-même. Pour cette philosophie, nomade, avide de célébrer des alliances et de dire « oui » au devenir, la différence n'a rien à voir avec une différenciation d'avec autrui, qui nous exposerait à des schèmes d'opposition et à leur lot de dérives. Il s'agit d'une différence d'avec soi-même, non comme désagrégation ou évanouissement constant (ce qui distingue sa notion de devenir de celle d'Héraclite), mais comme vertige et moteur créateur. On penserait aussi à une optique sartrienne, pour laquelle la mobilité identitaire est précisément ce qui permet de nouer des relations vivantes et authentiques, sans déchirures ni domination: l'identité libre, n'est-ce pas justement de ne pas être ce que l'on est, et être ce que l'on n'est pas? Seules les choses sont bornées à être ce que qu'elles sont ou à ne pas être ce qu'elles ne sont pas...

Cette identité dans la différence d'avec soi de la belgitude, si on accepte de la faire se rencontrer avec la direction deleuzienne, spécialement bien mise en évidence par Alliez (1996 : 243-264), est riche, et au-delà de toute notion de divorce d'avec la référence parisienne, même si c'est en réaction à des discours jugés trop centripètes autour de l'inauguration du Centre culturel de la Communauté française de Belgique dans la Ville lumière que la notion a d'abord été utilisée. Et il en va de davantage que d'une "revendication de non identité », ou de «dire que l'on n'est rien », comme semblent le suggérer Denis et Klinkenberg (2005: 229). Cette suggestion est peut-être à relier à la qualité de ce dernier d'être signataire du Manifeste pour la culture wallonne (1983), lequel constitue un moment de réplique vive à la notion de belgitude. Le débat se joue certes à un niveau très fin, parce que cette dernière reste avant tout un thème poétique, alors que le Manifeste s'exprime sur le terrain politique au nom d'un ancrage territorial précis. Parmi les autres critiques explicites, notons l'objection d'être un refuge rhétorique (Degn, 1999) ou le surinvestissement de l'identité dans la langue, dont la magie permet précisément d'être partout et nulle part à la fois. Un tel surinvestissement entraînerait ou se fonderait sur une omission de l'hétérogénéité du monde francophone belge. Une paix royale,

XLinguae, Volume 13 Issue 1, January 2020, ISSN 1337-8384, eISSN 2453-711X 
tableau intérieur de la Belgique d'après-guerre, se voit par exemple vivement accusée d'éluder le point de vue wallon (Fontaine, 2000), alors que les découpages fédéraux accentuent la division du monde francophone entre Bruxellois et Wallons, entre les résidents d'une ville de services et ceux d'une région aux caractéristiques propres que les premiers perçoivent volontiers comme flottant sur une industrie décadente.

A l'heure de la belgitude, la visée à définir les lettres belges comme espace autonome, entre autres par appui sur les nouvelles institutions culturelles, est notamment sensible dans le souci de tracer des rencontres et de cerner des fils de destin commun avec d'autres littératures dites "périphériques", soucieuses de se poser (Québec, pays africains, Suisse, ...). C'est dans cette optique que certains écrivains belges démontrent parfois des décalages explicites par rapport à tout académisme de la forme, et même une tradition d'irrévérence (par exemple chez Verheggen). Le souci de faire de Bruxelles un pôle culturel alternatif à Paris, dans un monde unilingue mais polyculturel, est particulièrement identifiable chez un théoricien comme Quaghebeur :

« Notre culture de sérail est maintenue dans cet état par sa sujétion de fait à une institution littéraire extérieure, sur laquelle nous ne pouvons peser, parce qu'elle nous domine et qu'elle appartient, -mais ce serait un autre sujet de débat- à une histoire foncièrement différente de la nôtre. L'institution parisienne suit ses intérêts propres, c'est bien logique. N'en déplaise à d'aucuns, nous ne sommes pas des écrivains français. » (Quaghebeur, 1996)

A nouveau, cette démarche de théorisation se défend de tout enrayage idéologique, et de s'exposer à ne récolter dans les œuvres qu'un sens préconçu sous des schémas rigides plus ou moins conscients. Il s'agit d'épouser au mieux le devenir créatif des choses et des écrivains, sans oublier les structures collectives qu'ils traversent, et de nourrir une dialectique riche et vivante avec d'autres espaces autonomes.

«A l'intérieur de cet unilinguisme polyculturel s'opérera de surcroît une salutaire redistribution des valeurs (...) Les différentes esthétiques, tout en étant profondément enracinées dans des histoires, ne pourront plus aussi indûment être mises au service de glorioles nationalistes et impérialistes. » (Quaghebeur, 1980 : 509)

\section{La Belgique, à la croisée des devenirs}

" A la fin, par une légère anticipation qui nous mène au-delà de ce siècle, le pays devient plus petit encore. Au point qu'on pourra se demander s'il a jamais existé. » (Introduction à Une paix royale)

Une paix royale ne s'embarrasse pas d'une considération extrême pour la forme, au point que l'humour qui vivifie le roman et le plaisir certain pris à la lecture n'affranchissent pas, par moments, de l'impression d'avoir affaire à une écriture «bavarde ». C'est toutefois par une motivation bien consciente : d'abord, le caractère décousu fait pleinement partie de la filature réaliste d'un pays où « tout ce qui est réel est toujours inventé », par ailleurs haut-lieu du surréalisme et de ses associations libres ; ensuite, comme le résume Magnette (1998:46) : «Pierre Mertens a écrit non sans dérision une histoire belge. Une fable grotesque, qui flirte avec la grossièreté de l'âme flamande, qui s'amuse à être irrévérencieuse ». L'écriture se présente comme un espace de tissage à partir de traces puisées dans une mémoire à la fois singulière et 
collective, et joue avec la disposition plus ou moins convenue du Belge à s'autodénigrer, pour baliser l'image d'une micro-nation à la fois échauffée et en déroute :

«(Bruxelles est) une ville où les choses chavirent et ne sont pas remplacées... Un théâtre... Des cinémas de quartier... Une taverne qui flambe et qu'on ne reconstruit pas (...). On pourrait conclure par là la fin du pays lui-même, ce pays où tout fout le camp... Vous voyez? » (p. 378).

Le narrateur est un homme au-delà de la quarantaine qui ressemble étrangement à l'écrivain, au point de lui partager son prénom et sa période de naissance : c'est Pierre Raymond. Son patronyme ne siffle pas loin non plus, mais parfois pour s'en distinguer d'autant mieux, de plusieurs références de la mémoire collective : l'ancien ministre français Paul Reynaud, sur lequel nous reviendrons, ou la Chanson des quatre fils Aymon (Desorbay, 2006: 226). Guide touristique à la réflexion plus voyageuse que profonde, il écrit vaguement des articles. Un jour, au milieu des tombes des pharaons, il décide de cesser son travail et de se centrer sur un récit de la Belgique. Si Mertens avait coutume de faire parcourir le monde à ses lecteurs, il semblera ici les envelopper dans des frontières restreintes, mais qui ne vont pas sans impliquer des éléments reliés au monde entier. Toutes les frontières sont, et cela est salutaire, faites de contours flous : fiction et réalité, je individuel et je collectif, roman national et familial, etc. La forme, en apparence paradoxale, couple le déploiement d'un pays représenté et un anéantissement de soi du personnage principal : c'est que la régression et une certaine dépossession intérieure vont de pair avec un effritement du réel et la dissolution des schémas arrêtés pour relancer toujours un rapport aux choses. Autant de caractéristiques de la belgitude, faites d'indiscernabilités, au sein d'un monde et d'une histoire indécise, ouverte.

Pierre Raymond se propose notamment d'écrire une série de reportages sur le roi Léopold III. Il déclare de cette enquête qu'elle est un roman ou une fable à sa maîtresse Joy ; qu'elle est, par contre, une chronique rigoureuse et réaliste, d'un type nouveau, auprès de son directeur d'édition. Ce travail parcourt une pluralité jamais clôturée de dimensions reliées à la Belgique, tissant une toile vive (si le terme n'évoque rien d'arrêté) qui englobe le roi Léopold III et ses passions ethnologiques en Amazonie, les conflits intercommunautaires et linguistiques, les souvenirs d'enfance, les champions cyclistes si présents dans la conscience collective belge, etc. L'objet vélo par exemple, constitue un puits de réminiscences en même temps qu'un événement déclencheur de l'entrée en histoire du chroniqueur, puisque ce dernier affirme avoir été renversé alors qu'il était enfant par la voiture d'un roi, et même de deux : Léopold III et son fils Baudouin. Est-ce un épisode réel ou une folle élucubration? Le chroniqueur se réserve le droit, éventuellement exercé, de mentir. Dans une réalité toute pénétrée et composée de labyrinthes intérieurs, le "vrai peut parfois ne pas être le vraisemblable », et c'est au romancier qu'il incombe de raconter l'histoire, cette « tragédie sur mesure », d'un pays où tout est inventé. Contrairement à la parole d'un historien prétendument neutre, celle du romancier n'a pas de comptes à rendre, même devant celle des rois.

«Pierre Raymond avait beau ne pas penser qu'il en écrivait un, de roman, que c'était autre chose, de ni plus ni moins digne, il avait été surpris, attristé qu'on se méfiât à ce point du genre romanesque (...) «Chère Joy, comment ne voyez-vous pas qu'il s'agit d'une histoire parfaite ? Parfois, j'ai l'impression de l'avoir de toutes pièces imaginée... S'il m'avait été donné de n'inventer qu'une seule fable, c'eût été celle-là ! J'ai le

XLinguae, Volume 13 Issue 1, January 2020, ISSN 1337-8384, eISSN 2453-711X 
sentiment d'être né avec elle, de l'avoir portée en moi toute ma vie. Cette geste lamentable et magnifique, dérisoire et sublime. (...) En fait, chère Joy, voici la seule histoire que je veuille, que je puisse, que je doive raconter. Elle efface toutes les autres, elle annule d'avance tout autre projet narratif. » (p. 289)

L'écriture de la chronique, qui ici assimile donc la vérité des faits avec celle de l'intériorité, mélange des genres aussi différents que l'historiographie, l'autobiographie, le feuilleton, le roman historique, la parodie, des rappels d'épopée, la prose poétique, la saga... Les deux premiers, toutefois, prédominent en même temps qu'ils se confondent, et Mertens veille à ce que certains éléments de l'Histoire soutiennent la vérité du récit autobiographique, de manière à ne pas s'enfermer dans le pur fantasme. La naissance de Pierre Raymond par exemple, est écrite dans le sens de "faire vérité »: elle est située dans le contexte réel de la théorie de la fission de l'uranium, du tableau de Chagall intitulé Les Mariés de la Tour Eiffel, de l'enlèvement d'une blanche Brésilienne par les Indiens yanoama, d'un séisme ayant lieu au Chili, de la victoire de Sylvère Maes au Tour de France, du couturier Lanvin qui présenta un masque à gaz assorti au manteau, de la division de la Pologne entre Staline et Hitler ( «j'arrivais juste à temps pour assister à une guerre qui, jusque là, n'avait été que "drôle" »), etc. (p. 17). De tels faits correspondent à la période de naissance de l'écrivain Mertens lui-même qui a révélé tardivement avoir été un enfant caché (sa mère étant juive), et dont les premières années «se sont déroulées, et refermées, autour d'un secret dramatique et cuisant » (p. 18).

\section{L'inflation des traces : "Tout est dans tout"}

«Il appartient aux grandes nations d'écrire l'Histoire. Il revient aux petites de conter, ça et là, quelques fables dont la morale est secrète (...)» (p. 287). La situation du roman est celle d'une Belgique n'ayant plus de parole qui en donnerait une perception directe et pleine. Au moins le pays n'est-il pas encagé dans un discours unilatéral et dominant, et c'est un tissage libre entre les traces les plus diverses, hasardeuses, qui va en donner la mémoire. Le temps ne sera plus décidé, irréversible, pas plus que le passé ne serait encore un donné ferme dénué de coexistences illogiques. Dans cette peinture de la Belgique, il en va comme d'une mémoire-monde au sens deleuzien (1985), dont les nappes virtuelles seraient parcourues par le narrateur qui les actualise sous des formes de vie et agencements nouveaux. D'ailleurs, comment mieux survivre au chaos qu'en s'érigeant à en être soi-même l'organisateur, qu'en en élaborant le récit dans une rencontre entre éléments intérieurs et extérieurs? Ce que l'on appelle communément le réel se voit dès lors substitué par un agencement d'objets, de souvenirs de tous types : abdication du Roi, chevelure rousse de sa deuxième femme qui annonce toute une mer de flammes, journaux d'époque, eaux refoulées de la Senne qui s'abattront bientôt sur Bruxelles, sales et monstrueuses, etc. Toutes les traces sont bonnes à prendre, à « unifier librement » et à combiner infiniment : ce petit pays en contient bien assez pour encore faire monde.

De même que le projet du chroniqueur n'est jamais clôturé, il n'est pas non plus excluant. Il met par exemple en œuvre une désacralisation saine : entre les photographies royales et les traces les plus humbles, entre les plages aristocrates et les stations de métro sales, il n'y a aucune hiérarchie, aucune exclusion selon le vrai, le faux, le sérieux, la fantaisie, la fidélité, etc.

« Comme elle a dû feuler, l'"immaculée Astrid", dans les bras de Léopold ! (p. 290) (...) Il faudrait, Monsieur, prendre en horreur aussi bien les légendes embellissantes que les 
calomnies délibérées... Casser la statue. Ne plus apercevoir que l'Homme. » (p. 300)

Citons quelques exemples de traces reliées par le chroniqueur :

La fascination, à peine estompée à l'âge adulte, pour le mythe cycliste de la Belgique :

«Je me rince l'oeil avec les cadres en carbone monocoque, les tubes colombus nivracom, les boîtes de pédalier, les couronnes de fourche, les casques futuristes, les paires de gants aux doigts coupés, les maillots Lugano en nylon fluorescent couleur massepain, framboise, menthe ou grenadine » (p. 223).

\section{Les journaux et chroniques :}

Les revues comme Gens de chez nous, Moeurs locales, Fêtes d'autrefois et de maintenant et évoquant la Belgique (p. 387); les ragots journalistiques concernant la santé du Roi (p. 388); la chronique nécrologique où le narrateur lit la mort de beaucoup de personnes (p. 374); la chronique des faits divers (p. 372) ; les revues pornographiques du grand-père décédé, sa collection de femmes nues (p. 382). Les ruines de Bruxelles (p. 370) ... Un vieux film d'amateur montrant le Roi Baudouin lorsqu'il était jeune et grimpant sur un arbre (p. 391).

\section{Des œuvres plus ou moins littéraires, avec des traces d'intertextualité :}

Des poèmes faits par des versificateurs d'un jour à l'occasion de la mort du Roi Baudouin (p. 422); des livres comme Un barbare en Asie de Michaux, faisant partie de la collection du narrateur (p. 381). Un journal intime que Pierre Raymond écrivait quand il était adolescent (p. 353), des souvenirs relatifs à sa grand-mère et à sa roulotte, etc. Le lointain intérieur d'Henri Michaux; l'expression : «Ma foi, tout se passait bien, et dans le meilleur des mondes ?» (p. 383), qui est une référence au Candide de Voltaire ; l'expression : «Je préfère les saisons aux châteaux, si vous voyez ce que je veux dire! » (p. 384), qui est une référence à un poème d'Arthur Rimbaud ; « (...) et tutti quanti...» (p. 401).

\section{Des traces historiques :}

Certains espaces historiques sont décrits sous un mode littéraire et poétique : «Un château féodal à vingt kilomètres d'Ostende, dans lequel se sont retrouvés le roi Léopold et quatre ministres au moment des troupes allemandes (...) Elle (le château) est bâti, enfoui dans la roche même, on franchit une sorte de pont-levis en bronze, on s'engage dans un couloir éclairé en permanence (...) Le regard s'engloutit dans des gouffres d'une sauvagerie démente. » (p. 238).

Il s'agit d'un décor de théâtre réel, le metteur en scène étant l'Histoire elle-même...

XLinguae, Volume 13 Issue 1, January 2020, ISSN 1337-8384, eISSN 2453-711X 


\section{Des traces d'actualité :}

«(...) Le coup d'Etat contre Gorbatchev, la mort de Ceaucescu, la chute du Mur, l'arrivée au pouvoir de Fujimori, la division de la Tchécoslovaquie, la révolte des hémophiles contaminés par le sida, la compromission d'un homme politique socialiste dans la triche commise par un club de football, et tutti quanti..." (p. 400-401)

\section{Des traces évaporées :}

L'appartement de Pierre Raymond est un jour cambriolé, et l'inventaire des traces perdues ne fait qu'intensifier leur thème, car c'est d'un cambriolage de la mémoire, perdue pour toujours, qu'il s'agit :

«Les photos de Rebecca en monokini, une chanson, une reproduction, un livre sur la bataille du charbon (...) Une poignée de coquillages du genre "buccin" ou "trompette" (...) une chicote coloniale, un kieffieh palestinien, un maillot de trapéziste écaillé comme la queue d'une sirène, et si étroit qu'aucune élue de mon coeur ne réussit jamais à s'y glisser (...) Un fusil belge de type Herstal offert par un rebelle kurde. Un samovar afghan. La jupe plissée d'un athénien marchandée dans un bazar d'Ankara. Un oignon en or de la marque PatekPhilippe (...) Je me demande parfois, avec mélancolie : me ressemble-t-elle, cette collection d'hurluberlu " (pp.18-19). (...) Un ours en peluche, un très vieux soixante-dix-huit tours tout griffé, le calepin où un homme aurait noté, dans un code connu de lui seul sans doute, la magie passagère d'une heure espagnole... (p. 383)

Tout ce bric à brac dans les lieux de vie de Pierre Raymond, pénétrés d'énigmes intérieures et de mémoires belges, fait songer au désordre du bureau d'André Breton, dans lequel les objets s'agençaient comme les associations libres du rêve. Breton, qui aurait voulu voir les musées de son époque « organisés » comme tels.

\section{Et enfin, quelques envolées « romantiques » :}

(A Rebecca) « Fini le temps où, quand tu te penchais vers moi, mon cœur cognait contre les barreaux de sa cage, et où je me perchais sur la plus haute branche dans les jardins suspendus d'une juvénile espérance ! Où est passé le jeune tigre dont il ne reste que la faim qui l'assujettit ? Il savait bien que tu n'aurais pas de merci... Que le jour où ta vie t'aurait transportée à New Delhi, Obihiro ou Arequipa, quand je serais à Calcutta, à Osaka, à Montevideo, seule l'immensité de ce qui nous séparait nous rapprocherait encore... » (p. 325)

\section{La Belgique envahie et irréalisée par les eaux}

Dans une vision mêlant un sens apocalyptique et une poésie totale, délicieusement enfantine, le pays se trouve pris au piège d'un déluge dans la dernière partie. La présence des eaux ne va pas sans rappeler un élément très présent dans la mémoire littéraire belge : par exemple avec la figure esthétique du mythe du nord que déployaient les auteurs symbolistes de la fin du XIXe siècle, et dont les canaux, la brume et les aiguilles de pluie de la Bruges rodenbachienne constituent peut-être la 
manifestation la plus dense. Autre exemple, plus tardif, avec La comtesse des digues (Marie Gevers, 1931), dont l'héroïne doit harmoniser ses amours pour un fleuve et pour un homme.

Digues déchiquetées, littoraux égrugés, camions éventrés, trains disséqués,... Mertens investit un caractère visuel très partagé dans la littérature francophone belge, et dépeint un délire meurtrier de pluies et d'eaux en avalanches, remontantes et descendantes. Enfin, « qui parle ici de délire ? (...) Est-ce délire si la réalité passe l'imagination? » (p. 489). Dans un chaos tonnant, le pays, décrit comme un «trou triangulaire» (p. 480), ne peut que s'affaisser et être emporté dans son propre tourbillon. Ceinturée, noyée mais aussi masochiste, Bruxelles, dans sa parure de lieux signifiants, assiste à sa propre décomposition (p. 479). Les avertissements et les clignotements dans la brume (autre trace d'intertextualité), pourtant, n'avaient pas manqué. Mais on n'avait point voulu les entendre, comme " on s'obstine à ne pas entendre, la veille d'une invasion, les bruits de bottes aux frontières du pays » (p. 457) ; ce qui nous ramène à la période de naissance de Pierre Raymond. Aurionsnous habité un lieu qui ne méritait pas son bonheur? Toute renaissance sera difficile, car l'artisan fasciné par son propre crime se doit de l'expier plusieurs fois (p. 485).

« D'une manière générale, les habitants ont tendance à régresser, ils redeviennent comme des enfants, mais meurtriers, sanguinaires. Ils jouent à la guerre, mais pour de vrai. Des us et coutumes d'autres époques, des mœurs barbares, font, avec l'afflux de l'eau, leur réapparition. (...) Ils ne sont plus en quête que d'un langage dans lequel correspondre (...) On les entend s'interpeller d'un esquif à l'autre (...) Flibustiers d'une nation fantôme, boucaniers du rien, que pourront-ils devenir quand le pays ne sera plus qu'un boat people? Et si celui-ci allait s'éperonner, se rompre, une nuit (...), quel orchestre consentirait encore à jouer? » (pp. 483-484). Notre pays se divisait au moment même, ultime, où nous aurions pu nous réunir. Qui allait donc mourir le premier, du pays ou de nous ? Les paris étaient fermés. » (p. 487)

Les eaux atteignent à un engloutissement des formes et aux dessins obscurs, non sans laisser songer aux montées du brouillard sur la ville de Gand chez Franz Hellens, «comme une mer où sombraient une infinité de barques immobiles « (Hellens, En ville morte), ou aux envoûtements par les brumes de Paul Willems. Dans les mythes et images de l'histoire des hommes, l'eau est tour à tour présentée comme un élément ambivalent qui en même temps féconde, se donne, détruit, se refuse ... Par elle s'alternent le déluge et la régénérescence, la vie et la mort : «Il n'y avait pas de meilleure et pire ivresse qu'hybride ! Quelle baptismale et funèbre bordée ! ( (p. 459). La tempête sépare les villages et leurs habitants ; elle leur confisque leur histoire en même temps qu'elle pourrait susciter leur jubilation à se trouver réunis (p. 488). Tel un élément dans la philosophie de Bachelard, inéluctablement marqué d'ambivalence, l'eau ouvre la rêverie aux déploiements infinis, et l'entraîne dans une intimité profonde d'avec les choses.

«On eût dit que, partout, l'eau était devenue la norme et la terre d'exception (...) L'œil ne se lassait pas de contempler ces paysages où seuls les clochers, les têtes de grues, les pignons et les tuiles faîtières des toits, qui émergeaient, ça et là, de l'eau unanime, rappelaient qu'on avait naguère connu en ce

XLinguae, Volume 13 Issue 1, January 2020, ISSN 1337-8384, eISSN 2453-711X 
lieu une existence terrestre. Des bateaux semblaient voguer sur place dans la ramure des arbres. Il se dégageait de ces images une poésie insensée les frappant d'irréalité, une poésie née du bouleversement de toutes choses (...) (p. 464) (...) Ceux qui, ayant perdu leur pays et leur langue, leurs anciens points de repère, pensent tout recommencer sans doute à partir de rien. Mais au bout du voyage, il n'y a que l'eau qui les attend! Qu'en espèrent-ils donc, sinon l'enfouissement dans un songe tel qu'on peut en faire encore ici : opaque et sans couleurs ? (p. 484)»

Gaston Bachelard (1942 : 61-96) identifiait chez Edgar Poe une imagination habitée par les eaux stagnantes, et sales de par ce fait, parce qu'elles auraient comme thésaurisé les déchets et les mauvais rayons. Sans aller jusqu'à leur accorder un caractère aussi primordial chez Mertens, leur thème y est vivace, notamment dans la référence à la Senne. Rivière brimée au rang d'égout par les commerces, usines et brasseries de Bruxelles, la ville s'en préserve en la voûtant à partir de 1867 : on l'accuse de propager le choléra et autres maux, ses " eaux noires, empuanties, de stagner, de dormir... Que feraient-elles d'autre, écrasées sous tant d'opprobre ? Elles auraient comme honte de couler encore ; il ne leur reste qu'à raser les murs, qu'on les accuse de souiller au passage... » (p. 469). Il se pourrait d'ailleurs bien que l'eau sale renferme une violence, prête à tressauter pour catalyser le déluge : "Elle macère, croupit à toute allure: elle a la douceur de la pourriture»(p. 482). A nouveau, conformément à la définition bachelardienne de l'élément, l'image de l'eau stagnante n'est pas unilatérale: s'il ne faut s'embourber dans les «marais de la mort», elle participe aussi d'un lieu de l'autocritique (p. 205), qui peut se projeter comme force juge : « Comme pour châtier l'esprit même de division (...) On découvrait - trop tard qu'on s'engloutissait parce qu'on s'était répudié soi-même » (p. 487).

\section{Figure du roi Léopold III, rapport à la France et notion de « déshistoire »}

Nous avions souligné la non hiérarchisation des traces. On peut dire aussi que dans le roman de Mertens, les dimensions du petit et du grand s'entremêlent, à l'image des grandes réformes fédérales, qui cherchent à camoufler le rapetissement de l'unité nationale sous une incommensurable complexité... Lorsque Pierre Raymond se propose de mener une série de reportages sur Léopold III (1901-1983), l'histoire qui est contée, ou créée, devient celle d'un grand, mais qui passe pour un petit dans l'Histoire. Celui-ci est au centre de la plus grande crise ayant affecté l'État belge, celle de la Question royale, et d'une polémique qui lui est bien liée: celle de sa renonciation aux armes dix-huit jours après l'attaque allemande en 1940, jugée trop rapide par la parole courante de l'Histoire. Une paix royale fait du cas Léopold III le cœur métonymique d'une confrontation avec la France, autre tenant-lieu de la parole francophone, et réexamine le discours du ministre français Paul Reynaud, qui reprocha à la défection belge de précipiter l'attaque allemande sur l'Hexagone, en même temps qu'elle se privait des moyens d'être secourue par celui-ci.

Dans une étude aux accents lacaniens, Desorbay analyse l'impact et l'objectif de la fable de Mertens à «briser les effets » (2006: 231) des virulentes critiques de Reynaud, qui ont altéré et même tourné en dérision l'image de la Belgique dans l'historiographie, et ce en dépit de rétractations ou modérations plus tardives venant du côté français. Ouvrant la voie à une véritable «guerre des signifiants » (Desorbay, $2006: 221$ ), le discours du politicien masquait en réalité la faiblesse et les morcellements de la France elle-même, dont la force rhétorique ne suffira pas à la 
préserver. Une telle initiative n'est cependant pas elle-même innocente : Mertens est parfois oublieux de certaines nuances (Reynaud s'est défendu d'avoir utilisé le terme de «trahison» à l'endroit de Léopold), et « insère si habilement les signifiants incriminés dans son commentaire des citations reynaudiennes, que celui-ci trompera, autant que le discours lui-même autrefois, le lecteur non averti » (Desorbay, 2006 : 224). Cette initiative déchargerait aussi l'écrivain, par un glissement d'identifications, de l'enfant qu'il fut lui-même, appartenant à un clan hostile au roi : «La fougue de ses attaques anti-Reynaudiennes trahit (...) la force du sentiment de culpabilité qu'il dit ressentir vis-à-vis de Léopold III. » (Desorbay, 2006 : 229)

Le roman insuffle une version selon laquelle Léopold aurait surtout plié devant l'envahisseur par suivi de mauvais conseils et par naïveté, lui qui n'avait que peu de dispositions pour régner : " Pourquoi un pays qui ne croit pas en lui-même se serait-il donné un roi véritable ? (...) L'homme oublie qu'il est un roi - [il] n'aurait jamais dû le devenir sans doute» (p. 261). Investissant en Léopold des traits de grandeur, en contre-pied des frénésies rhétoriques, l'écrivain le crédite d'une passion plus profonde et sincère pour l'ethnologie, à laquelle il s'adonne après son retrait du trône en 1951. Il le crédite aussi d'une pensée noble à l'égard de l'altérité des peuples africains, et lucide contre les valeurs de la prétendue " civilisation ». Mertens est bien sûr conscient des oppressions et injustices commises par la colonisation belge, c'est-àdire d'une violence et de désirs secrets de majesté qui ne sont pas moindres que celles objectées aux propos de Reynaud.

« (...) Parfois, il se dit que s'il n'était pas appelé à régner, c'est ce qu'il aimerait le mieux faire : voir du pays. Il est allé une première fois au Congo, avant Astrid, pour étudier « l'état sanitaire des populations ", mais c'est plutôt leur état d'harmonie qui l'a frappé, leur résistance désespérée aux intrusions des Européens, des "civilisés », comme on dit; il prend photo sur photo (...), il se promet de revenir bientôt, de comprendre davantage, de photographier mieux » (pp. 184185). (...) Lui qu'on eût cru cramponné à son règne, qui sait s'il n'avait pas, de façon rusée, organisé lui-même et de longue date son évasion? Commis ce "péché contre l'État», dont d'aucuns l'inculpaient, pour changer de vie? Rendu à l'air libre, il était pris de vertige. La disgrâce qui sauve, ... qui y eût songé ? (p. 312) (...) Plaqué qu'il fut par son pays comme d'autres par une donzelle, le Roi avait voulu revenir à la contrée de toutes les origines » (p. 322).

Parmi les théoriciens ou écrivains reliés à la belgitude, et en dépit de la revendication d'un «non divorce ", la remise en question de la préséance parisienne sur la parole francophone prend parfois des tournures virulentes, qui ne constituent pas l'objet de cet article, pas plus qu'il ne serait question d'analyser le fond des débats sur Léopold III. On relèvera plutôt, avec le terme de " déshistoire », une intéressante disposition à introduire une brèche au sein des balises historiques, et à restituer ou à suggérer par le roman une polyphonie et des possibles. Certes, et pour reprendre l'exemple du discours de Reynaud, il est évident que la fable d'Une paix royale relève d'un genre différent par rapport au travail de l'historien ou au discours politique, du genre poétique par rapport au scientifique ou au rhétorique ; mais c'est justement de cette confrontation que la vérité instituée peut être nuancée, et qu'il sera éventuellement possible de rendre compte de certaines "paroles brisées ». C'est précisément par son errance et ses incertitudes intérieures que la belgitude se dote d'une force intempestive, dans des horizons qui dépassent volontiers son cadre

XLinguae, Volume 13 Issue 1, January 2020, ISSN 1337-8384, eISSN 2453-711X 
premier : Terre d'asile (Mertens, 1978) par exemple, se déroule aux confins de la Belgique et des problématiques du Chili de Pinochet.

Entraîné dans la régression de personnages dispersés, le lecteur assiste au déploiement d'un monde à l'image de la vérité de l'existence : le plus souvent imprévisible, décousue et bégayante, mystérieuse, dynamisée par l'imaginaire, etc. Une telle option ne peut qu'impliquer un bouleversement des formes classiques, de leurs ordres et enchaînements sécurisés. A l'image d'une Belgique en quête incessante de réinvention, et d'une irrésolution soigneusement célébrée, aucune vérité n'est définitive : «Je ne me réjouis pas peu de n'avoir pas trouvé le fin mot de toute cette incroyable histoire » (p.490), sont d'ailleurs les termes par lesquels Pierre Raymond termine le roman. Cette irrésolution, qui troue d'emblée toute tendance au grand récit (dans l'acception de Lyotard), à l'épopée, au fantasme de totalité ainsi qu'aux lignes d'arrivée dans l'ordre du temps, n'a toutefois rien d'un défaitisme ou d'une perte d'élan conquérant : Mertens décrit d'ailleurs la plupart de ses personnages comme portés par un instinct de survie, curieusement optimiste.

«Pour Mertens, écrire, c'est avant tout défaire: mais, le désordre du monde proclamé, les textes - en miettes le plus souvent- se constituent dans la poursuite effrénée et vaine d'un sens possible. D'où cet épanchement textuel, cet écoulement, qui désignent une dynamique dans laquelle insistances, répétitions, redondances, surcharges, collages et citations sont autant de possibilités d'expression vive, de mise en liberté du discours. » (Bajomée et Dorset, $1998: 14$ )

Cette vocation à la «déshistoire» par le roman, et le «creusement de l'aporie comme seule modalité de résolution des tensions »(Bergen, 1998: 90), impliquent une reconsidération de l'Histoire par la fiction et même d'indiquer, avec précautions et nuances, qu'elle revêt elle-même une grande part de fiction. Cette option ne va pas sans susciter certains questionnements : une certaine ingénuité créatrice et l'écoute attentive aux ouvertures du rêve peuvent avoir leur validité thérapeutique, mais sont-elles en tout cas et pour toute gravité de sujet, appropriées ? Car sous les cortèges d'associations, comparaisons, métaphores, et dans un traitement en forme globale de fable, le thème Léopold III par exemple, n'implique rien de moins que l'élaboration et la sublimation d'un trauma historique, que l'on retrouve fragmenté et rejoué par la subjectivité instable, farceuse et de son aveu potentiellement menteuse (p. 362), qu'est Pierre Raymond. L'opiniâtreté au décentrement et à la promotion d'autres ordres que logiques, qui prend volontiers des allures de plaisanterie (par exemple le burlesque d'un pays tiraillé entre son roi et un gouvernement en exil), ne constituent-elles pas autant d'évasions trop commodes ? On dira avec Bergen que : «La littérature est en droit de revendiquer un principe d'irresponsabilité en ce que l'auteur n'a pas à répondre de ses personnages, même si elle est comptable au regard de l'Histoire des possibilités de liberté ou d'oppression qu'elle a ouvertes dans le monde» (Bergen, 1998: 96). On rappellera en outre que l'assomption de la contingence implique également, à sa manière, une saine acceptation du manque. Aussi décidera-t-on ici de retenir et d'honorer surtout l'inspiration intempestive, ainsi qu'un sens de «l'entre-deux » ludique, vivifiant, que l'on va maintenant retrouver avec le personnage de Sciascia.

\section{Les rapports de l'Histoire et de la fiction dans la sicilitude de Sciascia}

Après avoir envisagé l'attribution de la parole historique au roman et la notion de déshistoire chez Mertens, nous allons à présent prendre des éléments de confrontation dans le roman Le Conseil d'Egypte, de Leonardo Sciascia (1963). Le choix de ce texte comme perspective de comparaison, qui ne peut certes ici qu'être 
suggérée, procède de plusieurs raisons. Tout d'abord, il engage une réflexion profonde sur les rapports de l'histoire et de la fiction : l'un des thèmes-clés, proféré par son personnage principal, est qu'il y a "plus de mérite à inventer l'histoire qu'à l'étudier". Ensuite, la sicilitude implique, comme la belgitude mais de manière très différente, toute la théorisation d'une condition existentielle au sein d'une aire "périphérique ", dans son cas géographiquement et naturellement plus facile à définir. Or, l'une des aspirations de la phase dite "dialectique» en littérature francophone de Belgique, malgré tout héritière de la non-évidence historique à se spécifier au vu de la proximité parisienne, de la Belgique-repoussoir de Baudelaire, etc., réside dans un souci de se confronter ou d'entrevoir des fils de destin partagé avec d'autres littératures périphériques (Denis et Klinkenberg, 2005: 223; Quaghebeur, 1980 et 1996). Mertens s'intéresse lui-même aux réalités périphériques, dont il voit une manifestation dans les vies de créateurs écartés ou persécutés politiquement, ou hérétiques, comme Sciascia lui-même. Ce dernier, écrivain lui aussi engagé, est décrit comme un inlassable chercheur de la vérité, dans un pays qui n'en a pas (Mertens, 1989 : 183196).

Comme le remarque Fichera, les périphéries culturelles sont parfois mieux à même de percevoir certaines évolutions historiques, mieux que les centres au sein desquels leur cours ou événement survient : "L'inactualité et le décalage socioculturel peuvent parfois tourner à l'avantage d'un point de vue qui, parce qu'il n'est pas enveloppé dans le déroulement des faits, se révèle étonnamment clairvoyant » (2017: 9). Cette lucidité n'implique pas pour autant qu'à partir de récits et exposés liés aux réalités régionales, il soit si évident de se poser en littérature universelle (on ne dira pas « littérature majeure», dont le terme nous enfermerait dans un schéma de lecture « littérature dominante-dominée »). A côté de cette clairvoyance, la conscience d'être périphérique peut se voir articulée à un sentiment de dévalorisation, spontané et partagé dans les cas de la belgitude et de la sictilitude, même s'il devient rapidement et surtout un stimulant, et même si les causes en sont différentes : "Comment peut-on être sicilien? », dira un mandataire de la Couronne de Naples dans Le conseil d'Egypte. Au niveau francophone belge, la réalité d'une représentation négative, même si elle se voit dépassée immédiatement, est également palpable. On en entend même le thème chez les théoriciens les plus en vue : Quaghebeur déclara que le début de son activité fut marqué par la prise de conscience que «le discrédit qui entourait cette littérature n'était pas fondé » (1996: 7). En matière d'universalité, nous avions notamment entrevu dans la mouvance de la belgitude une capacité à entrer dans une dialectique riche avec d'autres réalités ou espaces littéraires; chez Sciascia, il sera plus question d'une identité «bloc» et de « monde total », mais qui affirme aussi son universalité :

« Je demeure convaincu que la Sicile offre la synthèse de tant de problèmes, de tant de contradictions non seulement italiens mais aussi européens, qu'elle peut constituer la métaphore du monde d'aujourd'hui. Suis-je encore, dans ces conditions, un écrivain sicilien ? Et qu'est-ce qu'un écrivain ? » (1979:127)

Il convient certes de préciser la différence des contextes. Si nous avions laissé la belgitude à la véhémence des pluies et à la montée des eaux (à moins que ce ne soit le "pays qui descende », dit Mertens (1995: 482)), la Sicile de Sciascia subit la violence d'un ciel trop bleu, même s'il se tourmente vite pour virer à l'orage, tout comme elle subit celle du sirocco « qui vous enferme la tête dans un étau de feu », et fouette puissament la terre en déplaçant les poussières sèches (1979:7). Sciascia ne sait pas nager : la mer lui est détestable, comme à tous ces villages qui lui tournent le dos, parce qu'elle amène les envahisseurs (1979 : 35). Dans Terre d'asile de Mertens

XLinguae, Volume 13 Issue 1, January 2020, ISSN 1337-8384, eISSN 2453-711X 
par contre, la mer est une question de survie, vecteur de possibles, pour s'en aller et revenir, encore et encore. La Sicile de Sciascia n'est pas un lieu qui « existe et n'existe pas» mais celui d'un ailleurs figé, bout du monde isolé de l'Histoire, vertige métaphysique et lieu d'une temporalité close, cyclique, obsessionnelle. L'identité sicilienne n'est pas pensée sur le mode de l'errance, bien au contraire : elle serait restée immuable malgré les envahisseurs successifs (Grecs, Romains, Byzantins, Arabes, Français, Espagnols, Italiens...). Et l'île n'est pas le lieu de réformes conciliantes ou de compromis incessants, mais celui de l'expression violente du pouvoir, dont le pire est peut-être celui qui émane de sa terre propre (Sciascia est un grand analyste des réalités mafieuses). Une telle vision, dite "gattopardesque", volontiers exaltée par la littérature et le cinéma, suscite une vive critique de la part des historiens depuis les années 1970 (Frétigné, 2009 : 9-17), ou l'ironie d'un Italo Calvino par exemple, qui objecta à Sciascia sa clé étrangement claire et infaillible, dans sa lecture d'une île prétendument mystérieuse (Giarrizzo, $1995: 27$ ).

\section{Résumé du Conseil d'Egypte, et conclusion par voie comparative}

Ce roman, qui a été remarquablement adapté par le cinéaste Emidio Greco (2002), dépeint la Sicile à la fin du XVIIIe siècle. Basé sur des événements réels et des personnages que Sciascia a contribué à redécouvrir, il présente la Sicile sous les traits d'un double raté de la France : au sein de celle-ci, la Révolution se produit, alors que sur l'île, si les énergies révolutionnaires sont bien présentes, elles échouent. La figure la plus insigne en est celle de l'avocat Di Blasi, fasciné par les Lumières françaises, et finalement brisé sous la torture et exécuté.

Le récit s'articule autour d'un personnage qui apparaît (et logiquement) au sein de ce contexte figé : l'abbé Vella, qui truque de vieux manuscrits arabes alors qu'il parle à peine cette langue. Il réussit à faire croire que ceux-ci donnent une autre version de l'histoire de la reconquête de la Sicile, menée par les Normands contre les Arabes au XIe siècle. Avec une force de persuasion remarquable, il affirme sur base de ses «humbles et honnêtes traductions » que les ancêtres des aristocrates siciliens n'ont, contrairement aux énoncés jusque-là pris en compte, jamais participé à cette reconquête. Les aristocrates prennent alors peur de cette nouvelle vérité, car c'est précisément de cette dernière qu'ils tirent et justifient leurs privilèges. Dans le camp opposé, le Vice-Roi envoyé par Naples, ouvert aux idées nouvelles, encourage vivement les «recherches» de l'abbé, qui promettent de rabaisser le pouvoir des barons. Ces derniers tentent de répliquer en convoquant des experts (notamment le Suédois Hager), que Vella parvient provisoirement à tromper. Il s'en tire habilement jusqu'à ce que, par peur de la contagion révolutionnaire, un nouvel envoyé de Naples ne cesse soudain tout soutien à Vella et n'insuffle un recroquevillement sur les idées anciennes. L'abbé sera alors dénoncé, tandis que le jeune révolutionnaire Di Blasi est exécuté.

Mertens décrit Sciascia sous les traits généraux et bien modernes d'un « enquêteur », qui met lui-même en scène des enquêteurs; à cette nuance près que l'écrivain est davantage conscient que la poursuite, toujours par bribes, d'une vérité, n'aura pas de fin (1989: 191). Dans Le Conseil d'Egypte, Sciascia se montre particulièrement " ironiste », jusqu'à " trafiquer, caviarder, revoir et corriger l'histoire elle-même » (Mertens, 1989: 190). Le besoin de fiction y est en effet singulièrement intense car au fond, l'idée-maîtresse du Sicilien est la suivante : dans le contexte d'une île qui ne conçoit l'avenir et les ordres sociaux que comme répétitions du passé, qui castre tout élan vers le futur, la seule alternative consiste à truquer les origines.

Il convient de nuancer les rapprochements : alors que dans Une paix royale, la déshistoire et les enquêtes imaginatives, voire fantaisistes, de Pierre Raymond, faisaient d'emblée de l'histoire une fiction, à l'image d'un pays dans lequel « tout ce qui est réel est toujours inventé », on observe que l'histoire et la fiction désignent a priori deux ordres différents chez Sciascia. A ceci près que la fiction créée par Vella, si 
elle ne réussit pas, au final, à interférer et à influencer réellement l'histoire, va révéler cette dernière comme étant elle-même une forme de fiction (mais qui suscite des effets certains). L'avocat révolutionnaire Di Blasi le résume en ces termes: «L'aventure de l'abbé Vella n'aurait pas été possible si l'histoire elle-même n'était une imposture ». Peut-être n'était-ce pas d'abord au Conseil d'Egypte que Mertens pensait en observant que l'histoire suit volontiers, vient comme "plagier» l'œuvre de l'écrivain sicilien, jusqu'à en « donner un peu de froid dans le dos » (1989: 186). Mais ce que suggère cette formule trouve une validité à l'intérieur du roman : derrière l'abbé apparaît directement une multitude de figures de faussaires qui s'emboîtent et se révèlent les unes les autres.

Sciascia a contribué à mettre en avant le caractère collectif de l'imposture (Cederna, 1999) : le pouvoir napolitain encourage les recherches de l'abbé par intérêt politique. Quant aux aristocrates, ils recherchent et financent l'intervention des experts, également par intérêt. Ces derniers, censés identifier le trucage du manuscrit et l'imposture des traductions sont finalement eux-mêmes aussi des faussaires: l'expert Hager par exemple, s'enferme dans une notion toute académique de reconnaissance des codes (son indiscutée science de la traduction), qui court-circuite toute disposition à la surprise et aux imprévisibilités créatrice de la vie. En outre, il corrompt sa science en la mettant au service de l'idéologie, car il n'est pas dupe des intentions des barons. Si l'abbé parvient à le tromper, c'est précisément parce qu'il le place devant un événement (ces mystérieuses traductions) qui perturbe ses schémas, son rabaissement de la créativité et de la connaissance à une extension de ce qu'il connaît déjà. Nous n'aurons la place ici que pour rappeler cette idée nietzschéenne selon laquelle l'homme qui cherche la « vérité », comme s'il s'agissait d'une substance ou forme bloquée, ne le fait jamais sans quelque mobile obscur, comme la vengeance ou l'opportunisme...

Enfin, puisque nous avions mis en avant le caractère ludique et irrévérencieux de Pierre Raymond, au sein d'un questionnement relatif à la pertinence de la fable ou d'un « principe d'irresponsabilité » (Bergen) en littérature, il convient de souligner un caractère comparable chez Vella, qui n'a d'autre mauvaise intention que celle du farceur et du joueur. Le lecteur ne manquera pas de relever le comique de certaines situations, notamment lorsqu'après avoir simulé le vol de « l'original » dans ses appartements, l'abbé assiste à sa recherche à partir de la «traduction», les références échangeant ainsi leurs faces. Dans une optique nietzschéenne bien relayée par Deleuze (1985: 151-192), on dira aussi que l'abbé incarne l'innocence d'un devenir, et que c'est pour cette raison qu'il n'est au final ni jugeable ni jugé, contrairement à l'idéaliste Di Blasi. En outre, et de même que Pierre Raymond se fait auteur, Vella est un créateur : en ce sens, il incarne une figure complexe de faussaire, supérieure à toutes les autres. D'emblée il ne se limite pas à une simple transposition ou réitération des formes, mais agit en véritable artiste de la calligraphie, voire de l'enluminure. Il rejoint même, d'une certaine manière, la perspective nietzschéenne d'un art de la grande santé, dynamique des "puissances du faux » et de la "lutte contre la vérité $»$ : sa brisure du passé sicilien et du cortège de justifications sociales qui lui sont associées suggère bien, ne fût-ce que sur un mode passager, de nouvelles formes de vie.

Même si elles ne sont ici que suggérées, ces directions peuvent annoncer la possibilité d'un travail plus large de comparaison, avec l'expression de la belgitude que nous avons rencontrée. Et sans nous dissimuler les implications politiques que peut recouvrir ledit thème de la belgitude, la profondeur qu'on en retiendra pour l'instant est celle de ce vitalisme inventif, qui entend sublimer les régimes figés et se nourrir de ses propres décalages ou paradoxes, pour rêver de rivages qui « existent et n'existent pas ».

XLinguae, Volume 13 Issue 1, January 2020, ISSN 1337-8384, eISSN 2453-711X 


\section{Bibliographic references}

ALLIEZ, E. 1998. Sur le bergsonisme de Deleuze. In : Gilles Deleuze. Une vie philosophique (dir. E. Alliez). Le Plessis-Robinson : PUF/Institut Synthélabo, pp. 243264. ISBN 2-84324-022-0

BACHELARD, G. 1942. L'eau et les rêves. Essai sur l'imagination de la matière. Paris: José Corti. ISBN 2-7143-0032-4

BAJOMEE, D. - DORSET, M. 1998. Avant-propos. In: Pierre Mertens. La vérité de la fiction. s.l.: Ambedui, pp. 11-15.

BERGEN, V. 1998. L'écriture comme composition du chaos et travail de deuil. Questions sur l'écriture et l'identité. In: Pierre Mertens. La vérité de la fiction. s.l.: Ambedui, pp. 85-100.

BURNIAUX, R. - FRICKS, R. 1980 [1973]. La littérature belge d'expression française. Paris: PUF. ISBN 2130362834

CEDERNA, C. A. 1999. Imposture littéraire et stratégies politiques: Le Conseil d'Egypte des Lumières siciliennes à Leonardo Sciascia. Paris: Honoré Champion. ISBN 2745301012

DEGN, I. 1999. Histoire et fiction dans Une Paix Royale. In : La Vie Wallonne, Tome LXXIII, décembre 1999, pp. 45-71.

DELEUZE, G. 1968. Différence et répétition. Paris: PUF. ISBN 978-2-13-058529-9

DELEUZE, G. 1985. Cinéma 2. L'image-temps. Paris: Minuit. ISBN 2707310476

DENIS, B. - KLINKENBERG, J.-M. 2005. La littérature belge. Précis d'histoire sociale. Bruxelles: Labor. ISBN 978-2-87568-041-9

DESORBAY, B. 2006. Une paix royale de Pierre Mertens. Les histoires belges: grandeurs et misères du rapport à l'Autre de l'énonciation gallienne. In: Les écrivains francophones interprètes de l'Histoire. Entre filiation et dissidence (dir. B. Chikhi et M. Quaghebeur). Bruxelles: Peter Lang, pp. 217-232. ISBN-10: 90-5201-038-2, ISBN-13: 978-90-5201-0380

FICHERA, G. 2017. Engagement, fiction, vérité: Pasolini, Kalisky, Sciascia, Mertens. Macerata: Quodlibet. ISBN 978-88-229-0068-5

FRETIGNE, J.-Y. 2009. Histoire de la Sicile. Paris: Fayard. ISBN-10: 2213631549 , ISBN-13: 978-2213631547

FONTAINE, J. 2000. Le discours antiwallon (anti-démocrate) de Pierre Mertens (dans "Une paix royale"). In: Culture et société. La revue Toudi, n²5-26, février-mars 2000.

GIARRIZZO, G. 1995. La Sicilia di Sciascia. In: Cinema e letteratura: Leonardo Sciascia (a cura di S. Landi). Pordenone: Cinemazero, pp. 23-33.

KRALIK, R. 2013. The Reception of Soren Kierkegaard in Czech Language Writings. In: Filosoficky casopis, vol. 61, n. 3, pp. 443-451. ISSN 0015-1831.

MAGNETTE, P. 1998. L'homme contre la politique. Le roman conçu comme acte politique. In: Pierre Mertens. La vérité de la fiction. s.1.: Ambedui, pp. 39-48.

MERTENS, P. 1989. L'agent double. Bruxelles: Complexe. ISBN 2-87027-291-X

MERTENS, P. 1995. Une paix royale. Paris: Seuil. ISBN 2.02.023802.0

QUAGHEBEUR, M. 1980. Littérature et fonctionnement idéologique en Belgique francophone. In: La Belgique malgré tout (éd. J. Sojcher). In: Revue de l'Université de Bruxelles. 1-4/1980. Bruxelles: Université libre de Bruxelles, pp. 501-525.

QUAGHEBEUR, M. 2006. Anthologie de la littérature française de Belgique. Entre réel et surréel. Bruxelles: Racine. ISBN 2873864338

QUAGHEBEUR, M. 1996. Une arche inachevée. Un témoignage de Marc Quaghebeur sur l'institution littéraire belge depuis 1980. In: Textyles. Revue des lettres belges de langue française, $n^{\circ} 13 / 1996$, pp.137-148.

SCIASCIA, L. 1979. La Sicile comme métaphore (trad. M. Padovani). Paris: Stock. ISBN 2234010055

SCIASCIA, L. 1976. Le conseil d'Egypte (trad. J. De Pressac). Paris: Denöel. ISBN 2253013986 
SOJCHER, J. 1980. La Belgique malgré tout (éd. J. Sojcher). In: Revue de l'Université de Bruxelles. 1-4/1980. Bruxelles: Université libre de Bruxelles.

ZOZULAK, J. - VALCO, M. 2018. Byzantine Philosophy of the Person and its Theological Implications. In: Bogoslovni Vestnik, vol. 78, n. 4, pp. 1037-1050. ISSN 1581-2987.

Words: 9521

Characters: 59465 (33,04 standard pages)

Julien Delvaux, M.A, M.A, M.A.

École supérieure des Arts Saint-Luc Liège

Boulevard de la Constitution, 41

4020 Liège

Belgium

delvaux.julien@saint-luc.be 